The Journal of Symbolic Logic

Volume 31, Number 4, Dec. 1966

\title{
MEETING OF THE ASSOCIATION FOR SYMBOLIC LOGIC
}

A meeting of the Association for Symbolic Logic was held at the Waldorf-Astoria Hotel, New York City, on Monday, April 4, 1966 in conjunction with the American Mathematical Society. On April 5, 6 and 7, there was a Symposium on Mathematical Aspects of Computer Science jointly sponsored by the Association for Computing Machinery, the Association for Symbolic Logic and the American Mathematical Society, and supported financially by the Air Force Office of Scientific Research, the Institute for Defense Analyses, and the U.S. Army Research Office-Durham. The Symposium was presented in four sessions as follows:

Session I - Computation with symbolic and algebraic data.

Session II - Numerical methods for computers.

Session III - Software systems; mechanical linguistics, computer analysis of language.

Session IV - Theory of automata; artificial intelligence.

An invited address was presented by Professor Hilary Putnam entitled, Constructible sets and predicative hierarchies. In addition nine papers were delivered, and six were presented by title; the last six abstracts below were those presented by title.

Martin Davis

Yiannis N. Moschovakis. Hyperanalytic predicates.

We use the notation and terminology of S. C. Kleene's RFI and RFII (Recursive functionals and quantifiers of finite types I and II, Trans. Amer. Math. Soc., vol. 91 (1959) pp. 1-52 and vol. 108 (1963) pp. 106-142 respectively).

Let $\mathfrak{a}$ be a list of objects of types $1,2,3, \mathfrak{c}$ a list of variables of types $\leqq 3,{ }^{3} \mathbf{E}$ the type-3 object that represents function quantification (RFII 11.16). A predicate $P(c)$ is hyperanalytic in $\mathfrak{a}$, if it is recursive in the list ${ }^{3} \mathbf{E}, \mathfrak{a} ; P(\mathfrak{c})$ is $r . e$. in $\mathfrak{a}$, if for some $e$,

$$
P(\mathfrak{c}) \equiv[\{e\}(\mathfrak{a}, \mathfrak{c}) \text { is defined }] \text {. }
$$

(1) There exists a hyperanalytic number-theoretic predicate which is not recursive in any of the predicates $H_{a}^{2}(\alpha)\left(a \in O^{2}\right)$ of RFII, 11.27.

(2) Main theorem. For each list $\mathfrak{a}$ we define a set $N$ and to each $z \in N$ we assign a countable ordinal $|z|_{\mathbf{c}}$ and a predicate $\mathbf{G}_{z}(u, \alpha, v, \beta)$ such that the following conditions hold.

(a) $N$ is r.e. in $\mathbf{3} \mathbf{E}$, a.

(b) Each $\mathbf{G}_{\mathbf{z}}$ is hyperanalytic in a, uniformly for $z \in N$.

(c) If $P(\mathfrak{c})$ is hyperanalytic in $\mathfrak{a}$ and $\mathfrak{c}$ contains only variables of types $\leqq 1$, then $P(\mathfrak{c})$ is recursive in some $\mathbf{G}_{\boldsymbol{z}}$.

(d) If $|z|_{\mathfrak{c}}<|w|_{c}$, then $\mathbf{G}_{\boldsymbol{z}}$ is recursive in $\mathbf{G}_{\boldsymbol{w}}$, but $\mathbf{G}_{\boldsymbol{w}}$ is not recursive in $\mathbf{G}_{\boldsymbol{z}}$.

(e) A countable ordinal $\eta$ is equal to some $|z|_{\mathbf{c}}(z \in N)$ if and only if $\eta$ is the ordertype of some well-ordering of the natural numbers which is hyperanalytic in a.

The construction is in two steps:

(I) An elementary characterization (in the form of an inductive definition) of functions and functionals recursive in a list $\mathfrak{a}$ of objects of types $\leqq 3$.

(II) An extension to functionals with arguments of types $\leqq 3$ of several results that R. O. Gandy has proved for functionals with arguments of types $\leqq 2$, the most important of which is the following: there is a partial recursive functional $v\left(\alpha^{3}, c, e\right)$ such that

$$
(\mathrm{E} x)[\{e\}(\mathfrak{c}, x) \text { is defined }] \rightarrow\{e\}\left(\mathfrak{c}, v\left({ }^{3} \mathbf{E}, \mathfrak{c}, e\right)\right) \text { is defined. }
$$

We list some of the more interesting side results and corollaries: 
(3) A predicate $P(\mathfrak{c})$ is r.e. in ${ }^{3} \mathbf{E}$ if and only if there is an analytic $R(\mathfrak{c}, \mathbf{F})$ such that

$$
\begin{aligned}
P(\mathfrak{c}) & \equiv(\mathbf{E F}) R(\mathbf{c}, \mathbf{F}) \\
& \equiv(\mathbf{E F})[\mathbf{F} \text { is hyperanalytic in } \mathfrak{c} \& R(\mathfrak{c}, \mathbf{F})] .
\end{aligned}
$$

(4) A predicate $P(\mathbf{c})$ is r.e. in ${ }^{3} \mathbf{E}$ only if there is an analytic $Q(\mathfrak{c}, \mathbf{F}, \alpha)$ such that

$$
\begin{aligned}
P(\mathfrak{c}) & \equiv(\alpha)(\mathbf{F}) Q(\mathfrak{c}, \mathbf{F}, \alpha) \\
& \equiv(\alpha)(\mathbf{F})[\mathbf{F} \text { is hyperanalytic in } \alpha, \mathfrak{c} \rightarrow Q(\mathfrak{c}, \mathbf{F}, \alpha)] .
\end{aligned}
$$

((1) and (2) strengthen Kleene's XXVIII of RFI, the Representation Theorem for $\{e\}(\mathfrak{c}) \simeq w$ with $\mathrm{c}$ a list of variables of types $\leqq 3$.)

(5) A predicate $P(\mathfrak{c})$ is hyperanalytic if and only if both $P(\mathfrak{c})$ and $\bar{P}(\mathfrak{c})$ are r.e. in ${ }^{3} \mathbf{E}$.

(6) If $P(\mathfrak{c}, \alpha, \mathbf{F})$ is r.e. in ${ }^{3} \mathbf{E}$, then

$(\alpha)(\mathrm{EF})[\mathbf{F}$ is hyperanalytic in $\alpha, \mathrm{c} \& P(\mathrm{c}, \alpha, \mathbf{F})]$

$\equiv(\mathbf{E F})\left[\mathbf{F}\right.$ is hyperanalytic in $\left.\mathfrak{c} \&(\alpha) P\left(\mathfrak{c}, \alpha, \lambda \beta \mathbf{F}\left(\lambda t\left(2^{\alpha(t)} \cdot 3^{\beta(t)}\right)\right)\right)\right]$.

(7) There is a predicate $P(x, \alpha)$, r.e. in ${ }^{3} \mathbf{E}$, such that $(\mathrm{E} \alpha) P(x, \alpha)$ is not r.e. in ${ }^{3} \mathbf{E}$. (Received February $I$, Ig66.)

IRwin Mans. Probabilistic recursive functions.

The notions in the paper of de Leeuw, Moore, Shannon, and Shapiro, Computability by probabilistic machines (1956) are broadened, and extended to the class of all recursive probability distributions. Let $\Theta$ be the set of all finite sequences of $O$ 's and 1's, and $H$ be the set of all such infinite sequences. Define a partial order on $\Theta$ by $\theta_{1} \leqq \theta_{2}$ if $\theta_{2}=\theta_{1} * \theta$ (concatenation). Let $f: \Theta \rightarrow \Theta$ be (1) recursive, and (2) monotone nondecreasing. $f$ can be extended in a natural way to a function from $\mathrm{H}$ to $\mathrm{H} \cup \Theta$. Let $\mathrm{H}_{\theta}$ be the subset of $\mathrm{H}$ consisting of elements with initial segment $\theta$. Let $\psi$ be a probability measure on $\mathrm{H} . \psi$ is a recursive distribution if $\mathrm{H}_{\theta}$ is measurable for all $\theta$ and there is a recursive function $\mathrm{g}(\theta, \mathrm{n})$ such that $\left|\psi\left\{\mathrm{H}_{\theta}\right\}-\mathrm{g}(0, \mathrm{n})\right|<1 / \mathrm{n}$. For any $\theta$, and $0<p<1$, letting $a(\theta)$ be the number of 0 's and $b(\theta)$ the number of 1 's in $\theta$, a particular case of a distribution is $\psi_{\mathrm{p}}\left\{\mathrm{H}_{\theta}\right\}=\mathrm{p}^{\mathrm{a}(\theta)}(1-\mathrm{p})^{\mathrm{b}(\theta)}$. Lemma. $\psi_{\mathrm{p}}$ is recursive iff $\mathrm{p}$ is recursive. A pair $(\psi, \mathrm{f})$ will be called admissible if $\psi$ is a probability measure on $H$ and is recursive, $f: H \rightarrow H \cup \Theta$ is recursive and monotone, and $\psi\left\{\mathrm{x}\{\mathrm{f}(\mathrm{x}) \in \mathrm{H}\}=1\right.$. We will say that $\psi$ induces $\phi$ if, for all measurable subsets $H_{1}$ of $\mathrm{H}$ and $(\psi, \mathrm{f})$ admissible, $\phi\left\{\mathrm{H}_{1}\right\}=\psi\left\{\mathbf{f}^{-1}\left(\mathrm{H}_{1}\right)\right\}$. Theorem 1 . If $\psi$ is a recursive distribution on $\mathrm{H}$, and if $\psi$ induces $\phi$, then $\phi$ is a recursive distribution. Theorem 2. If $\phi$ is a recursive distribution on $\mathbf{H}$, then for $0<\mathrm{p}<1 \psi_{\mathrm{p}}$ induces $\phi$. Definition: $\mathbf{x} \in \mathbf{H}$ is called an atom of a distribution $\psi$ if $\psi\{\mathbf{x}\}$ is positive. Theorem 3. Every atom of a recursive distribution is recursive. These theorems circumscribe the class of calculations which can be accomplished through the availability of probabilistic methods. (Received April 1, rg66.)

CARol Karp. Applications of recursive set functions to infinitary logic.

Consider ordinary set-theoretical formulas with additional one-place predicate letter $\mathbf{A}$, individual constant $\mathbf{c}$. Such a formula is $\Sigma_{0}(\mathbf{A} ; \mathbf{c})$ if all of its quantifiers are restricted and is $\Sigma_{n}(\mathbf{A} ; \mathbf{c})\left(\Pi_{n}(\mathbf{A} ; \mathbf{c})\right)$ if it consists of a $\Sigma_{0}(\mathbf{A} ; \mathbf{c})$-formula with a string of $n$ alternating quantifiers beginning with $\exists(\forall)$. For a transitive class $T$, a given predicate $A$ on $T$ and set $c \epsilon T$, a $k$-place predicate $R$ is $\Sigma_{n}^{(T)}(A ; c)$ iff there is a $\Sigma_{\mathbf{n}}(\mathrm{A} ; \mathbf{c})$-formula $\varphi$ such that $\left(\forall \mathrm{x}_{1} \ldots \mathrm{x}_{\mathrm{k}} \in \mathrm{T}\right)\left(\mathrm{R}\left(\mathrm{x}_{1}, \ldots, \mathrm{x}_{\mathbf{k}}\right) \leftrightarrow F_{\left\langle\mathrm{T}, \varepsilon_{\mathbf{T}}\right\rangle} \varphi\left(\mathrm{A} ; \mathrm{c}, \mathrm{x}_{1}, \ldots, \mathrm{x}_{\mathbf{k}}\right)\right)$. Thus are obtained hierarchies of set-theoretical predicates analogous to Lévy's hierarchies of set-theoretical formulas in his $\boldsymbol{A M S}$ Memoir, \#57, 1965. It is implicit in the work of Takeuti that this hierarchy relative to $\mathrm{L}$, the class of constructible sets, is isomorphic to the hierarchy of ordinal predicates arising from the theories of ordinal computability of Takeuti, Machover, Levy, Kripke. The $\Sigma^{\left(L_{1}\right)} \cap \Pi_{1}^{(L)}$-predicates can be characterized either by schemata or by an infinitary Herbrand-Gödel computation 
system and a method of $R$. Jensen can be used to show that they are effectively isomorphic to the finitarily recursive ordinal predicates of Lévy. Relative to $H_{\omega}$, the hereditarily finite sets, the hierarchy is isomorphic to the ordinary arithmetical hierarchy with the $\Sigma_{1}^{\left(\mathrm{H}_{\omega}\right)} \cap \Pi_{1}^{\left(\mathrm{H}_{\omega}\right)}$-predicates corresponding to the recursive predicates of natural numbers.

Consider predicates Taut( $\mathrm{x})$, meaning $\mathrm{x}$ is a tautology (in the infinitary sense), and $\operatorname{Vld}(\mathrm{x})$, meaning $\mathrm{x}$ is a valid formula (in the infinitary sense). Thm. 1. (AC) Taut(x) is $\Pi_{1}$ and every $\Pi_{1}$-predicate is $\Sigma_{1}$ (Taut). Taut is therefore not $\Sigma_{1}$ (c) for any set c. Thm. 2. (AC) $\operatorname{Vld}(\mathrm{x})$ is $\Sigma_{2} \cap \Pi_{2}$ and is not a Boolean combination of $\Sigma_{1}$ (c)-predicates.

Let $\mathrm{H}_{\kappa}$ be the set of all sets hereditarily of power at most $\kappa$, where $\kappa$ is an infinite cardinal, and write $\Sigma_{1}^{\kappa}$ for $\Sigma_{1}^{\left(\mathrm{H}_{1}\right)}$. Let $\kappa$-Taut(x) and $\kappa$-Vld(x) be Taut and Vld restricted to $\mathrm{x} \epsilon \mathrm{H}_{\kappa}$. Thm. 3. (AC) (a) $\kappa$-Taut is $\Pi_{1}^{\kappa}$. (b) If $\kappa \exp \omega=\kappa$ then every $\Pi_{1}^{\kappa}$-predicate is $\Sigma_{1}^{\kappa}\left(\kappa\right.$-Taut). Thus $\kappa$-Taut is not $\Sigma_{1}^{\kappa}(\mathrm{c})$ for any $\mathrm{c} \epsilon \mathrm{H}_{\kappa^{*}}$ (c) (Cont. Hyp.) $\kappa$-Taut is $\Sigma_{1}^{\kappa}$ iff $\kappa=\omega$. (d) (Gen. Cont. Hyp.) $\kappa$-Taut is $\Sigma_{1}^{\kappa}(\mathrm{c})$ for some $\mathrm{c} \epsilon \mathrm{H}_{\kappa}$ iff $\operatorname{cf}(\kappa)=\omega$.

Proof and computation predicates have always been $\Sigma_{1} \cap \Pi_{1}$ and that situation will probably continue because a ZF-definable predicate that is functional and stable relative to transitive classes is always $\Sigma_{1} \cap \Pi_{1}$. We conclude from Thm. 3 that $\kappa^{-}$ propositional logic is not effectively axiomatizable (even allowing parameters) within $\mathrm{H}_{\kappa}$ if $\kappa \exp \omega=\kappa$. It is a theorem of D. Scott that $\kappa$-Vld is not $\mathrm{H}_{\kappa}$-definable. Therefore though valid $\kappa$-formulas can be proved from tautologies, there is in general no proof in $\mathrm{H}_{\kappa^{*}}$. Thm. 2 gives further information about systems of infinitary predicate logic.

Raymond M. Smullyan. Analytic cut.

We consider a Gentzen-type system $\mathrm{S}$ whose only logical inference rule is a cut rule, but weakened so that all proofs still obey the subformula principle. We first consider the postulates for propositional logic. Axioms: $\mathrm{X} \rightarrow \mathrm{X} ; \mathrm{X} \wedge \mathrm{Y} \rightarrow \mathrm{X} ; \mathrm{X} \wedge \mathrm{Y} \rightarrow \mathrm{Y}$; $\mathrm{X} \rightarrow \mathrm{X} \vee \mathrm{Y} ; \quad \mathrm{Y} \rightarrow \mathrm{X} \vee \mathrm{Y} ; \quad \mathrm{X} \vee \mathrm{Y} \rightarrow \mathrm{X}, \mathrm{Y} ; \quad \mathrm{Y} \rightarrow \mathrm{X} \supset \mathrm{Y} ; \quad \mathrm{X}, \mathrm{X} \supset \mathrm{Y} \rightarrow \mathrm{Y}$; $\rightarrow X, X \supset Y$. Rules (1) [Weakening] From $\Theta \rightarrow \Gamma$ to infer $\Theta^{*} \rightarrow \Gamma^{*}$ if all terms of $\Theta$ are in $\Theta^{*}$ and all terms of $\Gamma$ are in $\Gamma^{*}$; (2) [Analytic Cut] From $\Theta, X \rightarrow \Gamma$ and $\Theta \rightarrow \mathrm{X}, \Gamma$ to infer $\Theta \rightarrow \Gamma$, providing that $X$ is a subformula of some term of $\Theta$ or of $\Gamma$.

The above system $S_{0}$ is complete for propositional logic. If we add Gentzen's rules for the quantifiers we obtain a complete system for quantification theory. But we prefer to replace these rules by axioms as follows. Our formal objects shall be ordered pairs $\langle\sigma, \Theta \rightarrow \Gamma\rangle-$ which we write $\Theta \rightarrow_{\sigma} \Gamma$ - where $\sigma$ is a finite set of (individual) parameters (also called "constants"). [We informally read $\Theta \rightarrow_{\sigma} \Gamma$ as "for at least one value of $a_{1}, \ldots, a_{n}, \Theta \rightarrow \Gamma$ holds"']. We add to $S_{0}$ the axioms:

(1) $\forall \mathrm{x} \varphi \mathrm{x} \rightarrow \varphi \mathrm{a} ; \varphi \mathrm{a} \rightarrow \exists \mathrm{x} \varphi \mathrm{x}$; (2) $\varphi \mathrm{a} \rightarrow_{\mathrm{a}} \forall \mathrm{x} \varphi \mathrm{x} ; \exists \mathrm{x} \varphi \mathrm{x} \rightarrow \mathrm{a} \varphi \mathrm{a}$, providing a does not occur in $\varphi x$. We replace the structural and cut rules by: (1) From $\Theta \rightarrow_{\sigma} \Gamma$ to infer $\Theta^{*} \rightarrow_{\sigma} \Gamma^{*}$ (where $\Theta^{*}, \Gamma^{*}$ are as before); (2) From $\Theta, \mathrm{X} \rightarrow_{\sigma} \Gamma$ and $\Theta \rightarrow_{\sigma_{2}} \mathrm{X}, \Gamma$ to infer $\Theta \rightarrow \sigma \Gamma$, where one of $\sigma_{1}, \sigma_{2}$ is empty, and $\sigma$ consists of those parameters of the other which actually occur in $\Theta \rightarrow \Gamma$, (and again, X must be a subformula of $\Theta \rightarrow \Gamma$ ). This system $S$ is complete for first order logic (indeed, Gentzen's quantificational rules are easily derivable). (Received April I, rg66.)

Hidemitsu SAYEKI. Some consequences of normality of the space of models.

By $\mathrm{L}(\mu)$ we mean the first order predicate calculus with identity of a type $\mu . \mathrm{S}\langle\mu\rangle$ mean the class of all sentences in $L(\mu)$. We denote by $\widetilde{M}(\mu)$ the class of all interpretations of $\mathrm{L}(\mu)$ and by $\mathrm{M}(\mu)$ the family of all quotient classes of $\tilde{\mathrm{M}}(\mu)$ with respect to elementary equivalence in $L(\mu)$. Natural mapping will be denoted by $\sigma$. We introduce a topology into $\mathrm{M}(\mu)$ taking $\left\{\sigma \mathrm{X}^{*} ; \mathrm{X} \in \mathrm{S}(\mu)\right\}$ as open basis. Then $\mathrm{M}(\mu)$ is a compact Hausdorff space, whence is a normal space. Let $\mathrm{K} \subseteq \tilde{\mathrm{M}}(\mu) . \mathrm{K} \in \mathrm{EC}\left(\mathrm{EC}_{\Delta}\right)$ iff $\mathrm{K}=\sigma^{-1}(\mathrm{~F})$ for some clopen (closed) subset $\mathrm{F}$ of $\mathrm{M}(\mu)$. Suppose $\mu^{\prime} \supseteq \mu$. A mapping 
$\pi_{\mu}$ defined on $\mathrm{M}\left(\mu^{\prime}\right)$ is called $\mu$-reduction of $\mathrm{M}\left(\mu^{\prime}\right)$ iff it assigns to every element $a \mathfrak{M}$ of $\mathrm{M}\left(\mu^{\prime}\right)$ the element of $\mathrm{M}(\mu)$ whose representative is the $\mu$-reduct of $\mathfrak{M}$.

Theorem. $\pi_{\mu}$ is a continuous mapping of $\mathrm{M}\left(\mu^{\prime}\right)$ onto $\mathrm{M}(\mu)$ under which the image of each closed set is closed.

From the theorem follows that: $\mathrm{K} \in \mathrm{PC}_{\Delta}$ iff $\sigma \mathrm{K}$ is closed in $\mathrm{M}(\mu)$. Hence $\mathrm{K} \in \mathrm{PC}_{\Delta} \cap \mathrm{ACL} \Rightarrow \mathrm{K} \in \mathrm{EC}_{\Delta}$.

Let $\mu^{\prime} \supseteq \mu$. X $\in \mathrm{S}\left(\mu^{\prime}\right)$ is called definable in $\mathrm{L}(\mu)$ iff $\pi_{\mu}^{-1} \pi_{\mu}\left(\sigma \mathrm{X}^{*}\right)=\sigma \mathrm{X}^{*}$. Assume that $\alpha, \beta$ are disjoint sequences of predicates and that $L\left(\mu^{\prime}\right)$ is obtained from $L(\mu)$ by adjoining predicates of $\alpha, \beta$ (in symbol: $\mu^{\prime}=\mu+\alpha+\beta$ ). $\Sigma^{\prime}, \Sigma^{\prime \prime} \subseteq \mathrm{S}\left(\mu^{\prime}\right)$ are said to be prime modulo $\mu$ iff each sentence in $\Sigma^{\prime}, \Sigma^{\prime \prime}$ is definable in $\mathrm{L}(\mu+\alpha), \mathrm{L}(\mu+\beta)$ respectively.

Theorem. Let $\mu^{\prime} \supseteq \mu$. Assume that $\Sigma^{\prime}, \Sigma^{\prime \prime} \subseteq \mathrm{S}\left(\mu^{\prime}\right)$ are prime modulo $\mu$. If $\Sigma^{\prime} \cup \Sigma^{\prime \prime}$ is inconsistent in $\mathrm{L}\left(\mu^{\prime}\right)$, then there is an $\mathrm{X} \in \mathrm{S}(\mu)$ such that $\mathrm{X} \in \operatorname{Cn}\left(\Sigma^{\prime}\right)$ and $\Sigma^{\prime \prime} \cup\{X\}$ is inconsistent in $L\left(\mu^{\prime}\right)$.

In intuitionistic logic this generalization of Craig's Interpolation Theorem fails, since in a certain topology above theorem implies T4-Axiom, nevertheless the space of intuitionistic interpretations is $\mathrm{T}_{1}$-space, but not Hausdorff. (Received April 4, 1966. .)

Richmond $\mathrm{H}$. Thomason. A system of logic with free variables ranging over quantifiers.

The morphology of the system $Q$ resembles that of the classical predicate calculus with identity, except that $\mathbf{Q}$ is equipped with denumerably many quantificational variables $Q$, such that if $A$ is a wff then so is $(Q x) A$, for each individual variable $x$ and quantificational variable $Q$. Quantificational variables are interpreted as ranging over quantitiers, in the sense of Mostowski XXIII 217; this yields a natural generalization of classical satisfiability to Q-satisfiability.

Surprisingly, the ordinary Löwenheim-Skolem property fails for $Q$-satisfiability. E.g., let $\Theta_{1}=\left\{\sim x_{1}=x_{j} / i \neq j, i, j \in \omega\right\}, \Theta_{2}=\left\{F\left(x_{2 i}\right) / i \in \omega\right\}$, and $\Theta_{3}=\left\{\sim F\left(x_{21+1}\right) /\right.$ i $\epsilon \omega\}$. Let $\Gamma=\Theta_{1} \cup \Theta_{2} \cup \Theta_{3} \cup\{(Q x) F(x) \wedge \sim(Q x) \sim F(x)\}$. $\Gamma$ is readily Q-satisfied in a domain of cardinality $\boldsymbol{N}_{1}$, but is not $Q$-satisfiable in any domain of cardinality $\aleph_{0}$. (Under any $Q$-interpretation which satisfies $\Theta_{1} \cup \Theta_{2} \cup \Theta_{3}$ in a domain $D$, the extension $\mathrm{E}$ of $F$ must be infinite, as well as $\mathrm{D}-\mathrm{E}$. Then if $\mathrm{D}=\boldsymbol{\aleph}_{0}$, there must be a permutation of $\mathrm{D}$ taking $\mathrm{E}$ onto $\mathrm{D}-\mathrm{E}$; hence the truth-values of $(Q x) F(x)$ and $(Q x) \sim F(x)$ must be the same). This example is easily generalized to obtain a set $\Delta$ not Q-satisfiable in any domain of cardinality less than $\boldsymbol{\aleph}_{\omega}$.

Let the system $\mathbf{Q}$ have, in addition to standard axioms and rules for the classical predicate calculus with identity, all axioms having the form

$$
\operatorname{Per}(A(x, y), B(x), C(x)) \supset[(Q x) B(x) \equiv(Q y) C(y)] .
$$

where $\operatorname{Per}(A(x, y), B(x), C(x))$ is

$$
(x)(\exists ! y) A(x, y) \wedge(y)(\exists ! x) A(x, y) \wedge(x)[B(x) \equiv(\exists y)[A(x, y) \wedge C(y)]]
$$

and there are no free occurrences of $y$ in $C(x)$.

Conjecture: $\mathbf{Q}$ is semantically complete under the interpretation given above. We also suspect that proof of this conjecture would yield a Löwenheim-Skolem theorem for Q-satisfiability at the cardinal $\beth_{\omega} . \quad\left(\beth_{0}=\aleph_{0} ; \beth_{y+1}=2^{\Xi_{\nu}} ; \beth_{\omega}=\underset{i<\omega}{\beth_{1}}\right.$.) I.e., we conjecture that if a set $\Gamma$ of wffs is $Q$-satisfiable, then $\Gamma$ is $Q$-satisfiable in a domain of cardinality $\Xi_{\omega}$. Thus, assuming the generalized continuum hypothesis, this would be the best possible result. (Received April 4, I966.)

Donald J. Collins. A group with I8 defining relations and an unsolvable word problem.

Let $\mathrm{T}$ be an arbitrary Thue system and $\mathrm{V}$ a fixed word of $\mathrm{T}$. Then there exists a group $G(T, V)$, depending on $T$ and $V$ such that 
(1) if it is recursively unsolvable to determine for an arbitrary word $W$ of $T$ whether or not $W$ equals $V$ in $T$, then the word problem for $G(T, V)$ is unsolvable

(2) if $T$ has $m$ generators and $n$ defining relations, then $G(T, V)$ has $3 m+n+5$ defining relations.

If we specify $T$ as

$Z: s_{1}, s_{2}, \ldots, s_{m}$

$U: P_{j}=Q_{j}, j=1,2, \ldots, n$

then we present $G(T, V)$ as

$S: s_{1}, s_{2}, \ldots, s_{m}, k, t, q, a, b$

$D: a_{r}=s_{r} a \quad \quad b s_{r}=s_{r} b^{p+1} a^{p+1} \quad r=1,2, \ldots, m$

$\mathrm{s}_{\mathrm{r}}^{-1} \mathrm{q}=\mathrm{b}^{\mathbf{r}} \mathrm{bb}^{\mathrm{r}} \mathrm{qs}_{\mathbf{r}} \mathrm{b}^{\mathbf{r}} \mathrm{b}^{\mathbf{r}}$

$\mathbf{r}=1,2, \ldots, \mathrm{m}$

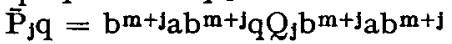

$\mathrm{j}=1,2, \ldots, \mathrm{n}$

at $=$ ta $\quad$ bt $=$ tb

$\mathrm{ak}=\mathrm{ka} \quad \mathrm{bk}=\mathrm{kb}$

$\mathrm{kV}^{-1} \mathrm{q}^{-1} \mathrm{tqV}=\mathrm{V}^{-1} \mathrm{q}^{-1} \mathrm{tqVk}$

where $p=m+n$ and $\bar{P}_{j}$ is the word obtained from $P_{j}$ by replacing each $s_{r}$ in $P_{j}$ by $s_{r}^{-1}$ (e.g. if $P_{j} \equiv s_{1} s_{2} s_{3}$, then $\bar{P}_{j} \equiv s_{1}^{-1} s_{2}^{-1} s_{3}^{-1}$ ).

G. S. Céjtin (Trudy Mat. Inst. Steklov, 52 (1958), 172-189) has specified a Thue system with 5 generators and 7 defining relations with the property that there is a word $\mathrm{V}$ of the system such that it is recursively unsolvable to determine for an arbitrary word $\mathrm{W}$ of the system whether or not $\mathrm{W}$ equals $\mathrm{V}$ in the system. (See also D. Scott (J. Symb. Logic, 21 (1956), 111-112)). Using the technique of M. Hall, Jr., (J. Symb. Logic, 14 (1949), 115-118) - since the Thue system under consideration possesses no relation of the form "non-empty word equals empty word", the simpler correspondence of "ith generator $\rightarrow \mathrm{abla}^{\mathrm{a}}$ " may also be employed - one can obtain a Thue system with 2 generators and 7 defining relations which has the same property. Consequently there exists a group with 18 defining relations whose word problem is unsolvable.

The group presented is a modification of that of W. W. Boone (Ann. of Math., 70 (1959), 209); the method of the proof is essentially that of J. L. Britton (Ann. of Math., 77 (1963), 16-32).

In Boone's presentation, the following typographical corrections should be made. The relation $q g_{\mathbf{1}}=g_{1} q$ should read $q g_{1}=d^{\mathbf{M}+1} c^{\mathbf{M}+1} a e^{\mathbf{M}+\mathbf{1}} d^{\mathbf{M}+1} g_{\mathbf{1}} q b^{\mathbf{M}+\mathbf{1}} c^{\mathbf{M}+\mathbf{1}} a e^{\mathbf{M}+\mathbf{1}} b^{\mathbf{M}+\mathbf{1}}$. The first assertion should read " $\ldots$ if and only if $t_{1} q W k W^{-1} q^{-1} t_{1}^{-1}$ equals $t_{2} q W k W^{-1} q^{-1} t_{2}^{-1}$ in $T_{\mathbf{P}}$ ").) (Received February I4, $^{2}$ 966.)

FREDERIC B. Fitch. A consistent modal set theory.

The proposed system of set theory is an extension of an underlying modal system with quantifiers and identity. The Barcan formula is assumed, and also unrestricted substitution for identity so that $[x=y] \supset \square[x=y]$. Set membership, $\varepsilon$, and unrestricted abstracts are added as primitive notions. The following principles are assumed:

$$
\begin{aligned}
& {\left[\begin{array}{lll}
x & \varepsilon & y
\end{array}\right] \supset \square\left[\begin{array}{ll}
x & \varepsilon y
\end{array}\right]} \\
& {\left[\begin{array}{lll}
x & \varepsilon \hat{y} \phi y
\end{array}\right] \equiv \square \phi x}
\end{aligned}
$$

The Russell paradox fails to arise because we get merely $[R \varepsilon R] \equiv \square \sim[R \varepsilon R]$, and therefore $\sim[R \varepsilon R]$ and $\diamond[R \varepsilon R]$. The system, indeed, can be shown to be consistent even if axioms of infinity and choice are added. The method of the consistency proof is similar to that for the system $\mathrm{C} \Delta$ of combinatory logic. (Received March II, 1966.) 
Trenchard MORE, JR. A strengthened Aussonderung schema.

The Aussonderung schema $\vdash \exists S \forall x . x \in S \Leftrightarrow, x \in A \& D^{-8}$ in ZF set theory is equivalent to $f J x D \Leftrightarrow \exists S \forall x, x \in S \Leftrightarrow D^{-8}$, where $J \times D$ is an abbreviation for $\mathbf{I S} \forall \mathbf{x} . \mathbf{D}^{-\mathbf{s}} \Rightarrow$ $x \in S$ and is read " $D$ is legal for $x$." $\mathbf{D}^{-\mathbf{s}}$ is any formula that has no free $\mathbf{S}$. If $\mathbf{a} ! \mathbf{x D}$ for $\exists x . D \& \forall y . D^{-y x} \Rightarrow x=y$, then $\vdash J x D \Leftrightarrow \exists ! S \forall x . x \in S \Leftrightarrow D^{-s}$ is an alternate Aussonderung schema. $D^{-y x}$ is got from $D^{-y}$ by substituting $y$ for all free $x$ where $y$ is free for $x$ in $D^{-y}$. If we permit UI and EG for terms of the form $\{x: D\}$, then $+\mathbf{\exists} ! \mathrm{S} S=\left\{\mathbf{x}: \mathbf{D}^{-\mathbf{s}}\right\}$. All terms exist and all individual variables are subject to quantification. With terms incorporated in the language, the Aussonderung schema may take the form $\vdash \mathbf{J} \mathbf{x D} \Rightarrow \mathbf{x} \boldsymbol{\epsilon}\{\mathbf{x}: \mathbf{D}\} \Leftrightarrow \mathbf{D}$.

If we assume the additional axiom schema $\vdash x \in\{x: D\} \Rightarrow d x D$, which is equivalent to $\vdash \neg \mathbf{J} D=\{\mathbf{x}: \mathbf{D}\}=\varnothing$ then the paradoxical sets are empty; $\vdash\{\mathbf{x}: \mathbf{x}\{\mathbf{x}\}=$ $\{\mathrm{x}: \mathrm{x}=\mathrm{x}\}=\left\{\mathrm{x}: \mathbf{D}^{-\mathrm{x}}\right\}=\varnothing$. The Aussonderung schema and the additional axiom schema are equivalent to a strengthened Aussonderung schema, $\vdash \mathbf{x} \in\{\mathbf{x}: \mathbf{D}\} \Leftrightarrow$. D \& $\rfloor \times D$, which simplifies the presentation of general set theory. The additional axiom schema has a partial converse $\vdash\{x: D\}=\varnothing \Rightarrow . D \Rightarrow \neg^{d x D}$. Although the more inclusive formula must be legal for the subset relation to hold, $1 \mathbf{J x B} \Rightarrow$. $\forall \mathbf{x}[\mathbf{A} \Rightarrow \mathbf{B}] \Rightarrow \cdot\{\mathbf{x}: \mathbf{A}\} \subseteq\{\mathbf{x}: \mathbf{B}\}$, there is no such restriction for equality $\vdash \forall \mathbf{x}[\mathbf{A} \Leftrightarrow \mathbf{B}] \Rightarrow\{\mathbf{x}: \mathbf{A}\}=\{\mathbf{x}: \mathbf{B}\}$. (Received February 14, 1966.)

Storrs MCCALI. How to make Boolean algebra Post-complete.

The classical system of Boolean algebra (BA) is incomplete in the sense that additions can be made to it without inconsistency. For example, the thesis $t a=0 \vee a=1$ can be added, resulting in a 'two-valued' algebra. This notwithstanding, BA can be made complete in such a way that it remains a genuine calculus of classes without becoming two-valued.

Consider the fragment (BI) of BA based on class-inclusion and the notions of the empty and the universal class alone. With the addition of a single non-classical rule of inference, this fragment can be shown to be Post-complete. The basis of BI is:

(i) Primitive symbols.

Class variables: $a, b, c, \ldots$

Class constants: 0,1

Connectives: $\cdot, \sim, \subset$

(ii) Rules of formation and definitions (usual).

(iii) Axioms.

1. Any set of axiom-schemata for two-valued propositional logic.

2. $(a \subset b \cdot b \subset c) \supset a \subset c$

3. $a \subset a$

4. $0 \subset a$

5. $a \subset 1$

6. $1 \notin 0$.

(iv) Rules of inference.

R1. Substitution for class variables.

R2. Detachment.

R3. If $\vdash x \mathbf{v} \alpha \subset \beta \mathbf{v} \gamma \subset \delta$, then either $\vdash x \mathbf{v} \alpha \subset \beta$ or $\vdash x \mathbf{v} \gamma \subset \delta$ (where $\alpha, \beta, \ldots$ range over class variables and constants, and $x$ is a zero- or many-termed disjunction of expressions of the form $\varepsilon \subset \zeta$ or $\varepsilon \notin \zeta$.)

Note that R3 is non-classical. If BA contained it, then the addition of (1) $\vdash a \subset 0 \vee 1 \subset a$ (making BA two-valued) would have the further result of either $a \subset 0$ or $1 \subset a$ being a thesis, which yields inconsistency. In the absence of (1), however, R3 may be consistently added. The proof is somewhat similar to Euka- 
siewicz's completeness-proof for assertoric syllogistic in Aristotle's Syllogistic, and yields both a decision procedure for BI and a proof of Post-completeness. (The role played by R3 is analogous to that of Słupecki's rule of rejection.)

$\mathrm{BI}$ is easily extended by the successive addition of primitive signs for class-complementation and intersection (resulting in the successive definability of 1 and 0 ). The addition of these yields an extension $\left(\mathrm{BA}^{+}\right)$of $\mathrm{BA}$ which is shown to be Post-complete by arguments similar to those for BI. It is noteworthy that $\mathrm{BA}^{+}$adds only new rules to BA, not new theses. (Received February II, I966.)

AlBert A. MUlin. Remarks on Hilbert's eighth class of problems.

This note gives some minor, but quite relevant, results bearing on a problem which for many analysts occupies a position similar to Fermat's Last "Theorem" for many algebraists. The crucial functions involved in the study are all effectively calculable. The crucial functions used are the "modified Liouville's function" and the "modified Möbius' function" as defined in Notices Amer. Math. Soc. 11 (1964), pg. 680 (64T-450). Lemma 1. Let natural number $\mathrm{n}$ be the cardinality of the following arbitrary finite index-sets of summations. Then $\mathrm{n}=\Sigma|\mu(\mathrm{m})|+\Sigma\left|\mu^{*}(\mathrm{~m})-\mu(\mathrm{m})\right|+$ $\Sigma\left|\lambda^{*}(\mathrm{~m})-\mu^{*}(\mathrm{~m})\right|$, where $\mu$ and $\lambda$ are the standard Möbius function and Liouville function, respectively. Lemma 2. $\Sigma\left|\lambda^{*}(\mathrm{~m})-\mu(\mathrm{m})\right|=\Sigma|\lambda(\mathrm{m})-\mu(\mathrm{m})|=\Sigma \mid \mu^{*}(\mathrm{~m})-$ $\mu(\mathrm{m})|+\Sigma| \lambda^{*}(\mathrm{~m})-\mu^{*}(\mathrm{~m}) \mid$. Based upon numerous analogies concerning properties of $\lambda$ and $\lambda^{*}$, and $\mu$ and $\mu^{*}$ together with partial numerical evidence the author Conjectures: (1) $\Sigma_{\mathrm{m} \leqq \mathrm{x}} \mu^{*}(\mathrm{~m})=\mathrm{o}(\mathrm{x})$ entails the prime number theorem, and (2) $\Sigma_{\mathrm{m} \leqq \mathrm{x}} \mu^{*}(\mathrm{~m})=\mathrm{O}\left(\mathrm{x}^{1}\right)$ entails the ordinary Riemann hypothesis for the ordinary zeta-function (see, e.g., S. Chowla, The Riemann hypothesis and Hilbert's tenth problem, Gordon and Breach, New York, 1965). Similarly, for the modified Liouville's function. (Received February 17, 1966.)

Richmond H. Thomason. On the semantical completeness of two systems of infinitary propositional calculus.

In a previous abstract appearing in this JOURNAL, the author presented systems $\mathbf{L} \mathbf{J}_{\infty}$ and $\mathbf{L S 4 _ { \infty }}$ of denumerably infinitary propositional calculus, corresponding respectively to intuitionistic logic and the modal logic $\mathbf{S 4}$. These systems have now been shown to be complete according to the criteria of validity formulated by Kripke for intuitionistic and modal logic.

The extension of Kripke's definition of validity to the infinitary case will be obvious to those familiar with Kripke's work. In our proof (which follows Henkin's proof of completeness for the classical predicate calculus) we use the notion of a $\mathbf{J}_{\infty}-\left[\mathbf{S} \mathbf{4}_{\infty}-\right]$ saturated set. A (perhaps nondenumerable) set $\mathrm{X}$ of wffs is $\mathbf{J}_{\infty^{-}}\left[\mathbf{S} \mathbf{4}_{\infty^{-}}\right]$satuarted if every denumerable subset of $\mathbf{X}$ is $\mathbf{J}_{\infty}-\left[\mathbf{S} \mathbf{4}_{\infty}-\right]$ consistent and if for all disjunctions $\mathrm{V} \Theta$ in $\mathrm{X}$, there is a wff $A$ in $\Theta$ such that $A$ is in $\mathrm{X}$. Using a (nonconstructive) argument of a familiar sort, we show that every $\mathbf{J}_{\infty}-\left[\mathbf{S} \mathbf{4}_{\infty}-\right]$ consistent set $\{A\}$ has a $\mathbf{J}_{\infty}-\left[\mathbf{S} \mathbf{4}_{\infty}-\right]$ saturated extension. Then, using an argument adapted from Henkin's work, we show that every $\mathbf{J}_{\infty}-\left[\mathbf{S} \mathbf{4}_{\infty}-\right]$ saturated set $X$ is satisfied under some interpretation (the interpretation being built out of the notation of $\mathrm{X}$ itself). Weak completeness theorems for the systems follow immediately from these results.

Algebraic analogues of these theorems can also be obtained, in the form of representation theorems for $\omega$-complete Brouwerian and closure algebras. (Received February $14,1966$.

JAmes C. OwINGS, JR. Some applications of metarecursion theory to $\Pi_{1}^{1}$-sets.

G. E. Sacks, in Metarecursive sets (this Journal, Sept. 1965) has proved that there exists a maximal $\Pi_{1}^{1}$-set and that every $\Pi_{1}^{1}$-set which is not $\Sigma_{1}^{1}$ is the union of two $\Pi_{1}^{1}$-sets which are not $\Sigma_{1}^{1}$. These facts prompted Hartley Rogers, Jr. to ask "Is the 
ordering of $\Pi_{1}^{1}$-sets under inclusion modulo finite differences isomorphic to the ordering of r.e. sets under inclusion modulo finite differences?" We can show that the answer is "no"; in fact, we show that the two orderings above and the ordering of the meta r.e. sets under inclusion modulo finite differences are all distinct. More precisely, we say $A$ is of type 1 if whenever $B$ is maximal in $A$ there is a maximal set $C$ such that $B=A \cap C$; otherwise, we say $A$ is of type 2 . It follows from a theorem of Lachlan that all maximal r.e. sets are of type 1 . We show that, whereas there exist maximal meta-r.e. sets of both types, all maximal $\Pi_{1}^{1}$-sets are of type 2 . The proof requires an extension of Lachlan's theorem and makes heavy use of the existence of two kinds of maximal meta-r.e. sets: those whose complements are bounded by some recursive ordinal and those whose complements are not.

In his doctoral thesis $G$. Driscoll proves that weak relative metarecursiveness (written $\leqq w$ ) is not a transitive relation on the meta-r.e. sets (see Metarecursive sets for definitions). Kreisel then asked if $\leqq w$ was transitive if restricted to $\Pi_{1}^{1}$-sets. The answer is no: we can construct $\Pi_{1}^{1}$-sets $A, B, C$ such that $A$ and $B$ are in the same metadegree and $\mathrm{B} \leqq{ }_{w} \mathrm{C}$ but $\mathrm{A} \coprod_{w} \mathrm{C}$.

We also lift Friedberg's 1-1 enumeration of the r.e. sets to metarecursion theory; i.e., there is a simultaneous 1-1 metarecursive enumeration of all meta-r.e. sets. We show that the corresponding result for $\Pi_{1}^{1}$-sets is false; i.e., there is no $\Pi_{1}^{1}$ predicate $\mathrm{P}(\mathrm{e}, \mathrm{n})$ such that for each $\Pi_{1}^{1}$-set $\mathrm{A}$ there is one and only one e such that $n \in A \leftrightarrow$ $\mathrm{P}(\mathrm{e}, \mathrm{n})$. In fact, it is impossible in metarecursion theory to give any simultaneous 1-1 enumeration of the $\Pi_{1}^{1}$-sets, even if all the recursive ordinals are used as indices. (Received February 25, 1966. .)

Raymond M. Smullyan. Uniform Gentzen systems.

In our paper [1] (A unitying principle in quantification theory, Proceedings of the National Academy of Sciences, June 1963) we hinted that our unifying $\alpha, \beta, \gamma, \delta$ notation should be useful in treating Gentzen-type systems. We now wish to show just how. For our present purposes, it is best to look at a Gentzen sequent $\Theta \rightarrow \Gamma$ as an ordered pair $\langle\Theta, \Gamma\rangle$ where $\Theta, \Gamma$ are finite sets rather than sequences of formulas. We now consider some systems which are uniform in the sense that the logical connectives and quantifiers never appear explicitly in any of the postulates, but only as hidden within the $\alpha, \beta, \gamma, \delta$ notation. What we called sentences in [1] we shall now call signed sentences (these are expressions $\mathrm{X}, \mathrm{X}^{\prime}$, where $\mathrm{X}$ is a closed formula of quantification theory). For the present, we make a strict syntactical distinction between $\mathrm{X}^{\prime}$ and $\sim \mathrm{X}$. For any signed formula $\mathrm{A}$, by its conjugate $\overline{\mathrm{A}}$ we mean the result of unpriming $A$ if $A$ is primed, or priming $A$ if $A$ is unprimed. Now, application (E) of [1] immediately yields a uniform system if we take $I(M)$ - henceforth written $\{M\}-$ to be the Gentzen sequent $M_{1} \rightarrow M_{2}$, where $M_{1}$ is the set of those (unsigned) sentences $X$ such that $X \in M$, and $M_{2}$ is the set of those (unsigned) sentences $Y$ such that $Y^{\prime} \in M$. [The reader can see concretely just what this system is by listing separately the 5 $\alpha$-cases, the $3 \beta$-cases, the $2 \gamma$-cases and the $2 \delta$-cases. The resulting system is almost standard].

The system of the above abstract is uniform, for the axioms can be subsummed under the following uniform schemata: Propositional Axioms: $\left\{\mathrm{X}, \mathrm{X}^{\prime}\right\},\left\{\alpha, \bar{\alpha}_{1}\right\}$, $\left\{\alpha, \bar{\alpha}_{2}\right\},\left\{\alpha_{1}, \alpha_{2}, \bar{\alpha}\right\}$, Quantificational Axioms: $\{\gamma, \overline{\gamma(\mathrm{a})}\},\langle\{\mathrm{a}\},\{\delta, \overline{\delta(\mathrm{a})}\}\rangle$, providing a does not occur in $\delta$.

The use of uniform notation is extremely handy and labor saving in treating the metatheory (e.g. in a constructing a finitary proof of Gentzen's Hauptsatz, 12 cases can be collapsed into 4). (Received April I, I966.)

Martin Zuckerman. Some theorems on the axiom of choice for finite sets.

Let $\mathrm{I}=\{$ integers $\geqq 2\}, \mathrm{I}^{*}=\mathrm{I} \cup\{1\}, \mathscr{P}=$ primes $\}$, and $\mathrm{C}=\mathrm{I}-\mathscr{P}$. If $\mathrm{S} \subseteq \mathrm{I}$ 
and $\mathrm{n} \in \mathrm{I}, \mathrm{S}_{(\mathrm{n})}$ will denote $\{\mathrm{x}: \mathrm{x} \in \mathrm{S} \& \mathrm{x} \leqq \mathrm{n}\}$. (For terminology, see $\langle\mathrm{I}\rangle \boldsymbol{F u n d}$. Math. 33 (1945), 137-168). Theorem. If $\mu(\mathrm{k})>\mathrm{p}$ for $\mathrm{N} \leqq \mathrm{k}<2 \mathrm{~N}$, then $\mu(\mathrm{n})>\mathrm{p}$ for all $\mathrm{n} \geqq \mathrm{N}$. If $\mathrm{n}$ is odd and composite, then $3 \leqq \mu(\mathrm{n}) \leqq \mathrm{n} / 3$; if, in addition, (1) $\mathrm{n}$ is not thrice a prime, then $3<\mu(\mathrm{n})<\mathrm{n} / 3$, or (2) $\mathrm{n}$ is thrice a prime, then $\mu(\mathrm{n})=\mathrm{n} / 3$. $\mu(\mathrm{n})>\frac{27}{16} \sqrt{\mathrm{n} / 2}, \mathrm{n} \in \mathrm{I}$. Theorem. If $[\mathrm{Z}] \rightarrow[\mathrm{n}]$ and if $\mathrm{m}=\max \mathrm{Z}$, then $\mathrm{n}<\frac{729}{512} \mathrm{~m}^{2}$; if $Z$ consists solely of composites or if $\max (Z \cap \mathscr{P})<\frac{8}{9} \mathrm{~m}$, then $\mathrm{n}<\frac{9}{8} \mathrm{~m}^{2}$. Theorem. $[Z] \rightarrow[n]$ implies that $Z$ contains a multiple of $n$ iff $n$ is prime. Theorem. For $n \in I$ and $p \in \mathscr{P}$, (1) $\left[\mathrm{C}_{(\mathrm{n})}\right] \rightarrow[\mathrm{p}]$ iff $\mathrm{p} \leqq \mathrm{n} / 2,(2)(\mathrm{M})$ is a NASC for the implication $\left[\mathscr{P}_{(\mathrm{p})}\right] \rightarrow[\mathrm{n}]$.

Definition. Let $\mathrm{m}_{\mathbf{i}}(Z)$ be the $\mathrm{i}^{\text {th }}$ smallest integer satisfying $\left[\mathrm{m}_{\mathrm{i}}\right] \rightarrow[Z]$, $\mathrm{i} \in \mathrm{I}^{*}$. Theorem. For $n \in I, m_{1}(\{n\})$ is prime iff $n$ is prime or $4 ; m_{i}(\{n\})$ is composite for $i \epsilon I$. A sufficient condition that $m_{1}(Z)=1 \mathrm{~cm}(Z)$ is that each element of $Z$ is square-free. For such sets, $m_{i}(Z)=i \cdot \operatorname{lcm}(Z)$ for all $i \in I^{*}$. All the $m_{i}(\{n\}), i \in I^{*}$, are determined for $2 \leqq \mathrm{n} \leqq 19$. Conjecture: $\mathrm{m}_{1}(\{\mathrm{n}\})=\mathrm{n}, \mathrm{n} \geqq 10$. Definition. An element $\mathrm{m} \in \mathrm{I}$ and a finite subset $Z \subset I$ satisfy $\left(M^{\prime}\right)$ iff for each $\mathbf{n} \in Z,\{m\}$ and $n$ satisfy $(M)$. Theorem. $\left(M^{\prime}\right)$ is necessary for the implication $[\mathrm{m}] \rightarrow[Z]$. $\left(\mathrm{M}^{\prime}\right)$ is a NASC for this implication in each of the following cases: (1) $\mathrm{m}<40$, (2) $\mathrm{m}$ is a prime power, (3) $Z=I_{(\mathrm{n})}, \mathrm{n} \in \mathrm{I}$, and (4) $Z=\mathscr{P}_{(\mathrm{n})}, \mathrm{n} \in \mathrm{I}$. Theorem. Let $\mathrm{m}, \mathrm{n} \in \mathrm{I}$. Let $\mathrm{p}(\mathrm{n})$ be the largest prime $\leqq \mathrm{n}$. Then $\mathrm{m}$ is a multiple of every prime $\leqq \mathrm{p}(\mathrm{n})$ iff $[\mathrm{m}] \rightarrow\left[\mathscr{P}_{(\mathrm{n})}\right]$ iff $[\mathrm{m}] \rightarrow\left[\mathrm{I}_{(\mathrm{n})}\right]$. Definition. For $m \in I, F(m)=\{n: n \in I \&[m] \rightarrow[n]\}$. Theorem. Each $F(m)$ is finite. $F(m)$ can be determined (1) for $\mathrm{m}$ a prime power $\mathrm{p}^{\mathrm{k}}$; if, in addition $\mathrm{m} \neq 2$ or $4, \mathrm{~F}(\mathrm{~m})=$ $\left\{\mathrm{p}^{1}: 1 \leqq 1 \leqq \mathrm{k}\right\},(2)$ for composite $\mathrm{m}<40$, (3) for certain composite $\mathrm{m}>40$. [m] iff $[\mathrm{n}]$ is false if $\mathrm{m}$ and $\mathrm{n}$ are distinct and at least one is a prime power other than 2 or 4.

Definition. Two finite disjoint subsets $Z_{1}$ and $Z_{2}=\left\{n_{1}, n_{2}, \ldots, n_{1}\right\}$ of I satisfy $(K)_{Z_{1}, Z_{2}}$ iff for every subgroup $\prod_{i=1}^{l} G_{n_{1}}$ of $\prod_{i=1}^{l} S_{n_{1}}$ such that no $G_{n_{k}}$ has fixed-points, $1 \leqq k \leqq 1$, there is a subgroup $H$ of $\left(\underset{i=1}{l} G_{n_{1}}\right)^{\omega}$ and a finite number $r$ of (not necessarily different) proper subgroups $K_{1}, K_{2}, \ldots, K_{r}$ of $H$ such that $\underset{i=1}{r} \operatorname{Ind}\left(H / K_{\dot{1}}\right) \in Z_{1}$. Obviously, $(\mathrm{K})_{\mathbf{Z}_{1}, \mathbf{Z}_{2}} \rightarrow(\mathrm{K})_{\mathbf{Z}_{1},\left\{\mathbf{n}_{1}\right\}} \&(\mathrm{~K})_{\mathbf{Z}_{1},\left\{\mathrm{n}_{2}\right\}} \& \ldots \&(\mathrm{~K})_{\mathbf{Z}_{1},\left\{\mathrm{n}_{1}\right\}}$. Theorem. If $(\mathrm{K})_{\mathrm{Z}_{1}, \mathrm{Z}_{\mathrm{a}}}$ is false, then there is a model for set theory (with urelements) in which $\left[Z_{1}\right]$ is satisfied, but for each $\mathbf{n}_{\mathbf{1}} \in Z_{\mathbf{2}},\left[\mathrm{n}_{1}\right]$ is unsatisfied. This extends $\langle 1\rangle$, Theorem 3. (Received April I, Ig66).

Herbert A. Simon. A note on almost-everywhere definability.

This paper develops a notion of almost-everywhere definability, first published in [1], that appears more suitable, for certain purposes of axiomatizing scientific theories, than the stronger notion of definability formalized by Tarski [2, Chapter X].

Let there exist a set of sentences $X^{\prime}$, and a subset $X, X \subseteq X^{\prime}$. Let $M$ be a set of models of $X$, and $\mu$ a measure defined on sets in $M$. Let $M^{\prime}$ be the corresponding set of models of $X^{\prime}$. Then $M^{\prime} \subseteq M$. Suppose that the measure, $\mu$, is such that $\mu(M)>0$, and $\mu\left(M-M^{\prime}\right)=0$. Let ' $a$ ' be an extra-logical constant that is definable, in the sense of Tarski, on the basis of $X^{\prime}$, is not definable on the basis of $X$, and does not occur in $X^{\prime}-X$. Then we shall say that 'a' is definable almost-everywhere in $M$ on the basis of $X$ and with respect to $\mu$.

The method of Padoa for showing that a constant is not definable in a theory cannot be used to show it is not almost-everywhere definable. In particular, in several axiomatizations of classical particle mechanics [3 and 4], the concept of mass, while undefinable in the sense of Tarski, is definable almost-everywhere.

[1] Simon, Herbert A., Definable terms and primitives in axiom systems, in Henkin, Suppes and Tarski (eds.), Symposium on the axiomatic method; Amsterdam, North-Holland Publishing Company, 1959 (pp. 443-453). 
[2] Tarski, A., Logic, Semantics, Metamathematics; London, Oxford University Press, 1956.

[3] Simon, Herbert A., The axioms of Newtonian mechanics, Philosophical magazine, Ser. 7, 33; 888-905 (1947).

[4] McKinsey, J. C. C., A. C. Sugar and Patrick Suppes, Axiomatic foundations of classical particle mechanics, Journal of rational mechanics and analysis, 2: 253-272 (1953). (Received November 5, I965.) 